\title{
BMJ Open Short-term and long-term case-fatality rates for myocardial infarction and ischaemic stroke by socioeconomic position and sex: a population-based cohort study in Sweden, 1990-1994 and 2005-2009
}

\author{
Ninoa Malki, ${ }^{1}$ Sara Hägg, ${ }^{1}$ Sanna Tiikkaja, ${ }^{2,3}$ Ilona Koupil, ${ }^{4}$ Pär Sparén, ${ }^{1}$ \\ Alexander Ploner ${ }^{1}$
}

To cite: Malki N, Hägg S, Tiikkaja S, et al. Short-term and long-term case-fatality rates for myocardial infarction and ischaemic stroke by socioeconomic position and sex: a population-based cohort study in Sweden, 1990-1994 and 2005-2009. BMJ Open 2019;9:e026192. doi:10.1136/ bmjopen-2018-026192

- Prepublication history and additional material for this paper are available online. To view please visit the journal (http:// dx.doi.org/10.1136/bmjopen2018-026192).

Received 31 August 2018 Revised 27 March 2019 Accepted 12 June 2019
Check for updates

\section{(c) Author(s) (or their} employer(s)) 2019. Re-use permitted under CC BY-NC. No commercial re-use. See rights and permissions. Published by BMJ.

For numbered affiliations see end of article.

Correspondence to

Ninoa Malki; ninoa.malki@ki.se

\section{ABSTRACT}

Objective Case-fatality rates (CFRs) for myocardial infarction (MI) and ischaemic stroke (IS) have decreased over time due to better prevention, medication and hospital care. It is unclear whether these improvements have been equally distributed according to socioeconomic position (SEP) and sex. The aim of this study is to analyse differences in short-term and long-term CFR for MI and IS by SEP and sex between the periods 1990-1994 to 2005-2009 for the entire Swedish population.

Design Population-based cohort study based on Swedish national registers.

Methods We used logistic regression and flexible parametric models to estimate short-term CFR (death before reaching the hospital or on the disease event day) and long-term CFR (1 year case-fatality conditional on surviving short-term) across five distinct SEP groups, as well as CFR differences (CFRDs) between SEP groups for both Ml and IS from 1990-1994 to 2005-2009.

Results Overall short-term CFR for both MI and IS decreased between study periods. For Ml, differences in short-term and long-term CFR between the least and most favourable SEP group were generally stable, except in long-term CFR among women; intermediate SEP groups mostly managed to catch up with the most favourable SEP group. For IS, short-term CFRD generally decreased compared with the most favourable group; but long-term CFRD were mostly stable, except for an increase for older subjects.

Conclusion Despite a general decline in CFR for Ml and IS across all SEP groups and both sexes as well as some reductions in CFRD, we found persistent and even increasing CFRD among the least advantaged SEP groups, older patients and women. We speculate that targeted prevention rather than treatment strategies have the potential to reduce these inequalities.

\section{INTRODUCTION}

Although global mortality rates for cardiovascular disease (CVD) have decreased by

\section{Strengths and limitations of this study}

- In this study, we use the entire Swedish population and a follow-up period of 20 years to analyse the association between socioeconomic position (SEP) and case-fatality rates (CFRs) in myocardial infarction (MI) and ischaemic stroke (IS).

- We modelled short-term and long-term CFRs separately, which allowed us to deal adequately and statistically correctly with the fact that almost $1 / 3$ of all deaths occurred within the first day after the event.

- With our statistical methods, we estimated both CFRs and CFR differences.

Information on risk factors and hospital care treatment before and after the event was not available, which prevented us from investigating medication mechanism for the association between SEP and CFRs for MI and IS.

- Socioeconomic status was based on Swedish population census information, which was more current at the beginning of the study period than at the end; however, sensitivity analyses indicate that this has little or no effect on our findings.

$22 \%$ over the last two decades, CVD is still the major cause of mortality and disability worldwide. ${ }^{1}$ This decline in CVD mortality can partly be attributed to decreasing CVD incidence, suggesting effective primary prevention and improvements in modifiable risk factors such as smoking, but is also due to reduction in CVD case-fatality, reflecting improved primary and secondary care. ${ }^{23}$

Healthcare in Sweden is largely tax-funded with a low out-of-pocket payment, a system that allows for equal access to healthcare irrespective of financial considerations. Still, there is consistent evidence that CVD case-fatality differs by socioeconomic position (SEP), ${ }^{4-7}$ 
with $50 \%$ worse prognosis for the lowest compared with the highest SEP group. ${ }^{8}$ Whether reductions in CVD case-fatality have been equitable between SEP groups, as well as between sexes, is an important public health question that can direct policy and healthcare towards more vulnerable groups; the importance of trend studies for assessing socioeconomic inequalities in CVD outcome has been emphasised previously. ${ }^{4}$

However, comparatively few studies have addressed how differences in CVD case-fatality between SEP groups change over time: Davies et al report persistent socioeconomic differences in short-term case-fatality for myocardial infarction (MI) in Scotland between 1988 and $2004^{9}$; Igland et al found persistent educational inequalities during the period 2001-2009 for MI case-fatality in Norway. ${ }^{10}$

Individuals with an acute CVD event who reach a hospital have access to a range of effective treatments that have contributed to observed improvements in CVD case-fatality. ${ }^{11}{ }^{12}$ A significant proportion of fatalities, however, occurs directly after an CVD event, almost $30 \%$ of patients with MI in Sweden died within 1 day after this event ${ }^{13}$ and the corresponding figure for stroke was $15 \%{ }^{13}$ Still, most studies of case-fatality in CVD exclude deaths outside hospitals ${ }^{89}$ often because of lack of data. This is an important limitation, as SEP may affect the chances of immediate and long-term survival in different ways.

The aim of this study was to analyse changes in shortterm and long-term case-fatality rates (CFRs) in MI and ischaemic stroke (IS) between the periods 1990-1994 and 2005-2009 by age, sex and SEP for the entire Swedish population.

\section{MATERIALS AND METHODS \\ Study population}

We used Swedish registers with high quality and coverage (online supplementary methods) and a follow-up period of 20 years. The Swedish National Patient Register (NPR) and the Swedish Cause of Death Register (CDR) were used to identify all cases of first primary MI and IS in two study periods, 1990-1994 and 2005-2009. All subjects who died from or were treated for MI or IS between ages 45 and 84 years at time of event were identified according to the International Classification of Diseases (ICD-9 and ICD-10, online supplementary table S1).

Sex and birth date of study subjects were retrieved from the Total Population Register. We followed study subjects from the date of MI/IS until death, emigration (based on the Swedish Migration Register) or end of follow-up 1 year after the disease event.

\section{Exposure}

We identified SEP from the mandatory Swedish Population and Housing Census in 1990; for subjects with missing information on SEP, we retrieved information from the 1980 census. SEP was derived from the
Socioeconomic Index ${ }^{14}$ (online supplementary methods), with five different categories, high non-manual (HN-M), low non-manual (LN-M), self-employed (SE) including farmers, high manual (HM) and low manual (LM). HN-M is supposed to be the most advantaged group and LM the least advantaged with LN-M, SE and HM as intermediate groups.

\section{Outcome}

Main outcomes are short-term mortality (defined as death within 1 day after an acute MI/IS event, including death outside the hospital) and long-term mortality (as 1-year survival, conditional on surviving the first day). For sensitivity analyses, we also considered 7-day, 28-day and 90-day survival, conditional on surviving the first day. As virtually all short-term fatalities (MI: 99.7\%, IS: 99.2\%) and great majority of long-term fatalities (MI: $89 \%$, IS: $84 \%$ ) are recorded as having CVD as primary or contributing cause of death, we study all-cause mortality as outcome.

\section{Statistical methods}

For both MI and IS, we modelled short-term and longterm case-fatality separately. All subjects were included in a logistic regression model for the binary outcome of dying before reaching the hospital or on the date of the disease event. Subjects who survived the first day of the disease event were included in a time-to-event analysis using flexible parametric models ${ }^{1516}$ for 1-year survival.

For both models, we included SEP as the main exposure of interest and adjusted for sex and age as a natural spline with three df. In total, we fit eight models for all possible combinations of outcomes (MI vs IS), shortterm and long-term case-fatality and period (1990-1994 vs 2005-2009). In each case, we started with a fully saturated model with a three-way interaction between age, sex and SEP and simplified by removing model terms that did not achieve statistical significance at a nominal level of $\alpha=0.05$, until no further simplification was possible. These final models were used to estimate the CFRs per 1000 subjects as either event probability (logistic regression, for short-term CFR) or 1-year survival probability (timeto-event analysis, for long-term CFR) as a function of age, sex and SEP. In both cases, estimates were calculated on the linear predictor scale, for all combinations of sex and SEP at three prespecified ages $(55,65,75)$ covering the age range under investigation; these contrasts were then transformed to the probability scale using the inverse link functions (logistic regression: inverse logit, flexible parametric survival regression: inverse log-log). Case-fatality rate differences (CFRDs) per 1000 subjects were estimated as differences in CFRs between SEP groups, with the most advantaged HN-M group as reference. For both CFRs and CFRDs, approximate standard errors were calculated via the multivariate delta-method ${ }^{17}$ and used to establish approximate 95\% CI.

For the time-to-event analysis, we selected time since disease event as time scale and modelled the corresponding baseline hazard as a natural spline function 
Table 1 Study cohort for case-fatality in myocardial infarction and ischaemic stroke by sex, age, socioeconomic position, short-term and long-term case-fatality in two periods 1990-1994 and 2005-2009

\begin{tabular}{|c|c|c|c|c|c|}
\hline & & \multicolumn{2}{|c|}{ Myocardial infarction } & \multicolumn{2}{|c|}{ Ischaemic stroke } \\
\hline & & 1990-1994 & 2005-2009 & 1990-1994 & 2005-2009 \\
\hline Total N & & 55456 & 72883 & 24046 & 53213 \\
\hline Female & & $11731(21 \%)$ & $23556(32 \%)$ & $7334(31 \%)$ & $22133(42 \%)$ \\
\hline \multirow[t]{4}{*}{ Age } & $45-54$ & $8036(14 \%)$ & $5770(8 \%)$ & $2402(10 \%)$ & 2537 (5\%) \\
\hline & $55-64$ & $15712(28 \%)$ & $17121(23 \%)$ & $5612(23 \%)$ & $9554(18 \%)$ \\
\hline & $65-74$ & $25911(47 \%)$ & $22023(30 \%)$ & $12491(52 \%)$ & $16306(31 \%)$ \\
\hline & $75-84$ & 5797 (10\%) & 27969 (38\%) & $3541(15 \%)$ & $24816(47 \%)$ \\
\hline \multirow[t]{5}{*}{ SEP } & LM & 18055 (33\%) & $23709(33 \%)$ & 7759 (32\%) & 17535 (33\%) \\
\hline & $\mathrm{HM}$ & $9633(17 \%)$ & $12581(17 \%)$ & 3699 (15\%) & $8178(15 \%)$ \\
\hline & SE & 8424 (15\%) & 8101 (11\%) & 3764 (16\%) & $5762(11 \%)$ \\
\hline & LN-M & 7757 (14\%) & $10650(15 \%)$ & $3681(15 \%)$ & $8472(16 \%)$ \\
\hline & HN-M & 11587 (21\%) & $17842(24 \%)$ & $5143(21 \%)$ & $13265(25 \%)$ \\
\hline \multirow[t]{2}{*}{ Case-fatality } & Short-term & 15726 (28\%) & 15425 (21\%) & 2152 (9\%) & 3562 (7\%) \\
\hline & Long-term & $2834(7 \%)$ & $4847(8 \%)$ & $1822(8 \%)$ & 4844 (10\%) \\
\hline
\end{tabular}

Study cohort: subjects are presented in numbers and percentage in two different periods 1990-1994 and 2005-2009, for myocardial infarction and ischaemic stroke. Age is categorised in 10-year intervals 45-54, 55-64, 65-74 and 75-84 years old at diagnosis. Short-term case-fatality is death within 1 day including death outside the hospital. Long-term case-fatality is death within 1 year conditional on surviving the first day. HM, high manual; HN-M, high non-manual; LM, low manual; LN-M, low non-manual; SE, self-employed; SEP, socioeconomic position.

with five df. The proportional hazard assumption for the effect of SEP on MI and IS was tested via an interaction term between SEP and time scale; as no statistically significant evidence for non-proportional effects at $\alpha=0.05$ was found in any of the models, these interaction terms were not retained.

Data preparation was carried out using SAS 9.4 software (SAS Institute, Cary, North Carolina, USA). STATA (Release 14. College Station, Texas, USA, Stata) was used for the statistical analysis.

\section{Patient and public involvement}

No patient was involved in this study.

\section{RESULTS}

\section{Myocardial infarction}

For MI, 55456 subjects aged 45-84 years old were identified in 1990-1994 and 72883 in 2005-2009 (table 1, online supplementary table S2). In both study periods, LM group had the highest CFR, and the HN-M had the lowest CFR across all three prespecified ages, among men and women (table 2).

Based on testing nested models, we retained only the main effects of the SEP, sex and age splines for study period 1990-1994, while for study period 2005-2009, we retained all two-way interactions (table 3 ).

\section{Short-term case-fatality rates}

For MI, 96\% of all short-term mortality was identified through CDR. Overall, short-term CFR for MI increased with age, with higher CFR in men compared with women.
Over time, the short-term CFR for MI decreased for all SEP groups at all three prespecified ages among men and women (table 2, figure 1).

CFRDs between LM and HN-M remained stable over the study period with a range of 39-66 extra deaths in 1990-1994 compared with 25-68 in 2005-2009 (per 1000 $\mathrm{MI}$ ) among men and women (online supplementary table $\mathrm{S} 3$, online supplementary figure $\mathrm{S} 1$ ).

In comparison, 55 years old men and women in the intermediate groups (HM, SE, LN-M) had substantial smaller short-term CFRD in 2005-2009 than 1990-1994; moreover, they managed to improve their survival and had non-significant CFRDs compared with HN-M. Men and women 65 and 75 years old in intermediate SEP groups did not improve their survival over the study period.

\section{Long-term case-fatality rates}

Long-term CFR for MI for those who survived first day increased generally overall with age and was slightly higher for men compared with women (table 2, online supplementary figure S2a).

For men, CFRD between LM and HN-M remained stable over the study period, with a range of 9-35 extra deaths per $1000 \mathrm{MI}$ in 1990-1994 compared with 11-35 in 2005-2009 (online supplementary table S3 and figure $\mathrm{S} 2 \mathrm{~b})$. For women however, CFRD between LM and HN-M seemed to increase, with a range of 7-29 extra deaths in 1990-1994 compared with 15-39 extra death in 2005-2009. For intermediate groups, CFRD seemed to decrease to similar levels as for HN-M, while there was an increasing tendency for women in LN-M. 
Table 2 Short-term and long-term case-fatality rates and 95\% confidence limits per 1000 subjects for myocardial infarction by socioeconomic position among men and women at prespecified ages 55, 65 and 75years old in 1990-1994 and 2005-2009

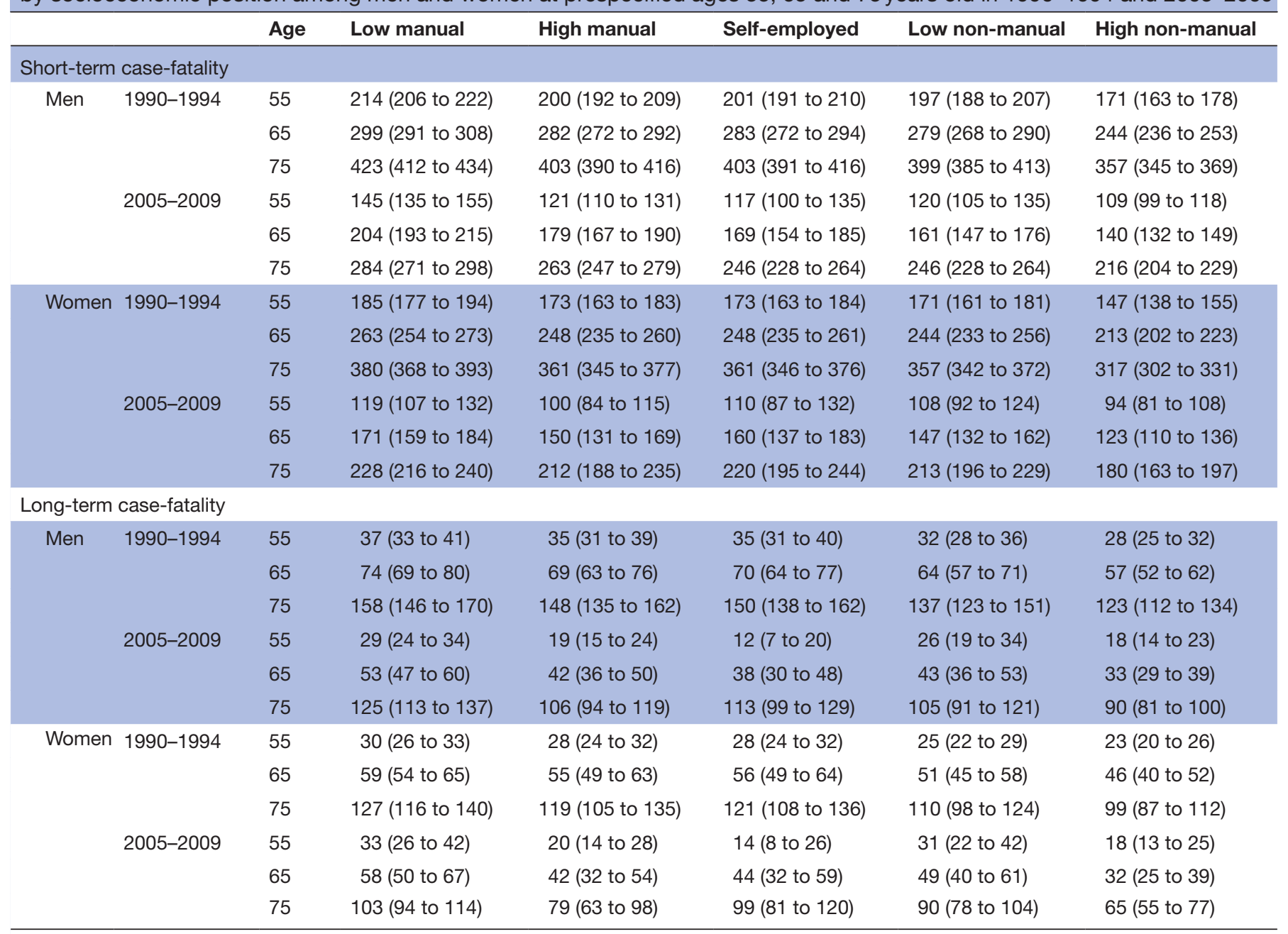

Age: prespecified ages 55, 65, 75 years old at diagnosis. Short-term case-fatality is death within 1 day including death outside the hospital. Long-term case-fatality is death within 1 year conditional on surviving the first day.

\section{Ischaemic stroke}

For IS, 24046 subjects aged 45-84 years old were identified in 1990-1994 and 53213 in 2005-2009 (table 1, online supplementary table S4). In both study periods, the LM group had the highest CFR and the HN-M had the lowest CFR across all three prespecified ages, both among men and women (table 4).

Based on comparison of nested models, we retained only the main effects of the SEP, sex and age splines for both study periods (table 3 ).

\section{Short-term case-fatality rates}

For IS, $68 \%$ of all short-term mortality was identified through CDR. Overall, short-term CFR for IS increased with age, with similar CFR among men and women. Over time, short-term CFR for IS decreased for all SEP groups, and at all three prespecified ages among men and women (table 4, figure 2).

Compared with HN-M, CFRDs for all other SEP groups decreased over time, among both men and women. For the LM group, the CFRD decreased from a range of 13-29 extra deaths per 1000 IS in 1990-1994 compared with 8-19 extra deaths in 2005-2009; for intermediate groups, the range decreased from 7-24 to 4-15 extra deaths per 1000 IS (online supplementary table S5 and figure S3).

\section{Long-term case-fatality rates}

Long-term CFR for IS ranged from 30 to 151 extra deaths in 1990-1994 compared with 25 to 116 in 2005-2009 (table 4, online supplementary figure S4a).

For men and women 55 and 65 years old, CFRD between the two study periods were stable (online supplementary table S5 and figure S4b). However, 75 years old men and women increased their CFRD from a range of 6-24 to 14-52 extra deaths per 1000 IS.

\section{DISCUSSION}

In this population-based cohort study, we found that shortterm CFRs for MI and IS have decreased from 1990-1994 to 2005-2009 for all SEP groups among both men and women. Short-term CFRs in the most disadvantaged LM 
Table 3 AIC and LRT $p$ values for evaluating candidate models for case-fatality in myocardial infarction and ischaemic stroke

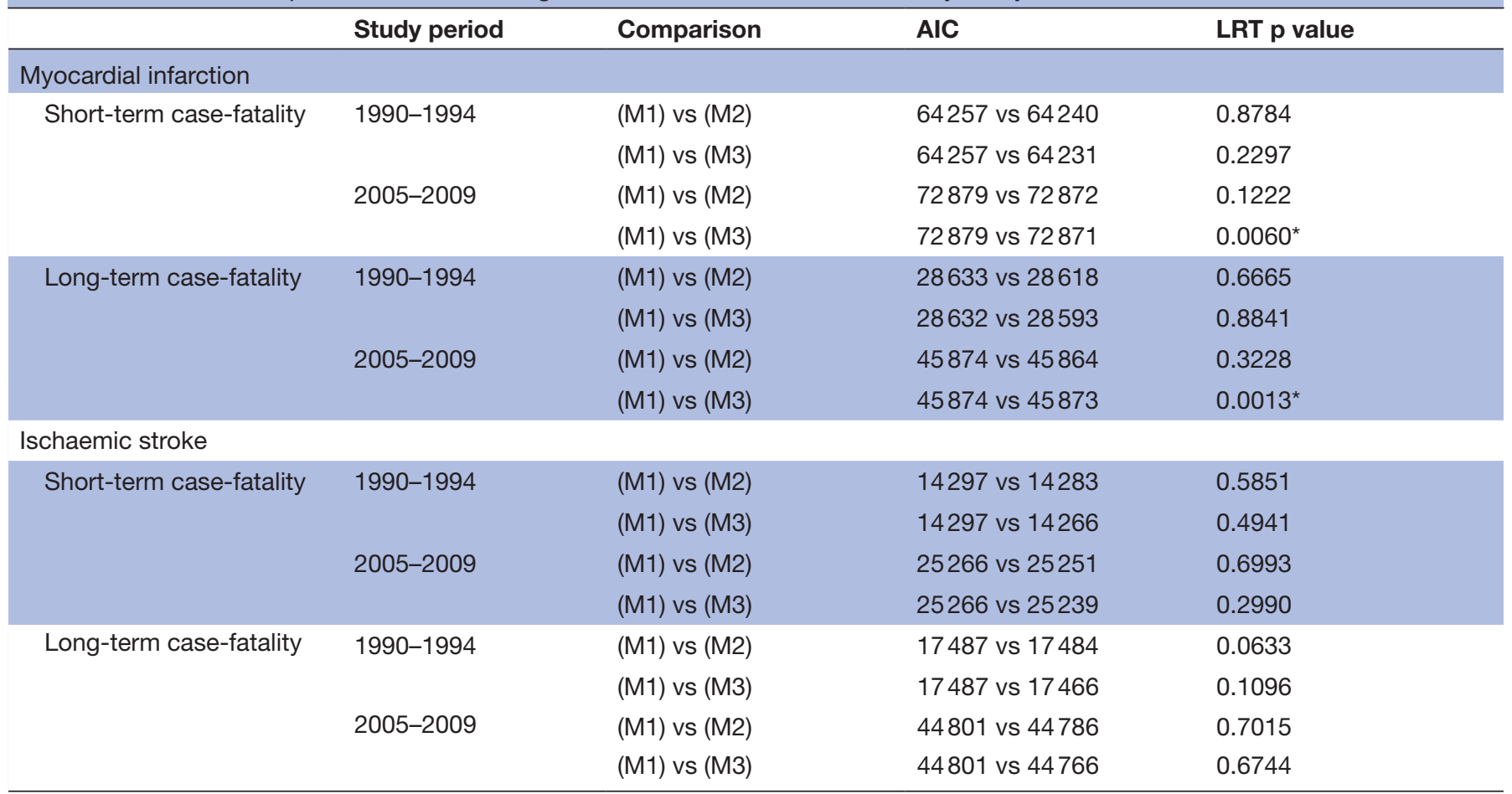

Age is included as a restricted cubic spline term with three df as main effect and in interaction terms.

Short-term case-fatality is death within 1 day including death outside the hospital. Long-term case-fatality is death within 1 year conditional on surviving the first day.

M1: SEP+Sex+ Age+SEP ${ }^{*}$ Sex + SEP $^{*}$ Age + Sex ${ }^{*}$ Age $+S^{*} P^{*}$ Sex ${ }^{*}$ Age

M2: SEP+Sex+Age+SEP*Sex+SEP*Age+Sex*Age

M3: SEP+Sex+Age

AIC, Aikaike information criterion; LRT, likelihood-ratio test.

group in 2005-2009 were lower than in the most advantaged HN-M group in 1990-1994. CFRDs for MI between the most and least disadvantaged LM and HN-M groups have mostly remained stable over time, but increased for long-term CFR among women. For IS, short-term CFRD

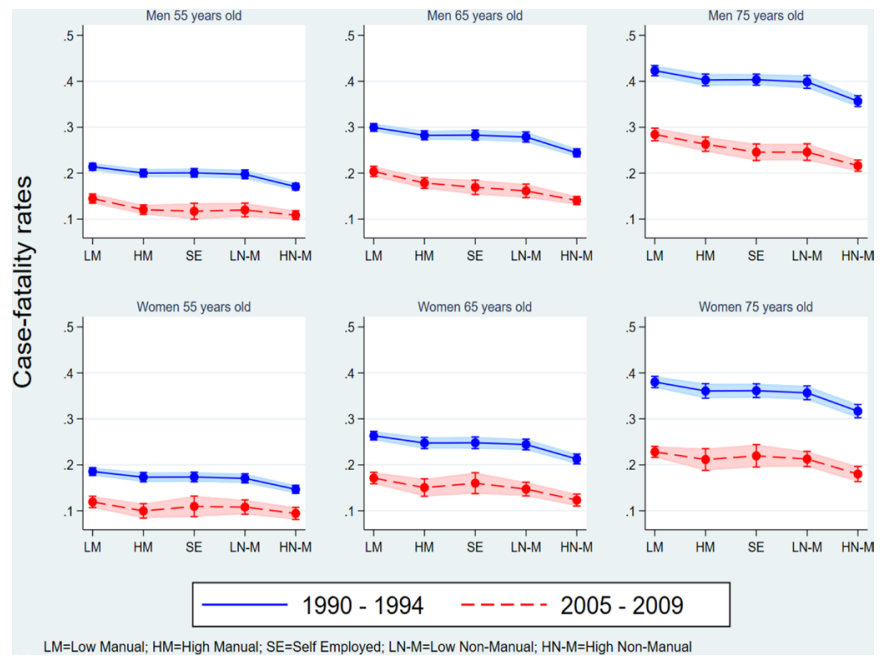

Figure 1 Short-term case-fatality rates in myocardial infarction by socioeconomic position for men and women at prespecified ages 55, 65 and 75 years old in 1990-1994 and 2005-2009. have generally decreased over time, while long-term CFRD among the oldest subjects (75 years) has increased.

General improvements in both short-term and longterm case-fatality for MI and IS have been reported in Sweden ${ }^{18-21}$ and other European countries ${ }^{22} 23$ as well as other high-income countries. ${ }^{24}$ Evidence for SEP inequality in both short-term and long-term CFR have been reported both in Sweden and globally. ${ }^{4}$ The degree with which these inequalities have changed given the temporal improvements is however less well understood, since comparatively few studies have reported inequalities in CVD case-fatality over time. Davies $e t a \ell^{\dagger}$ found persistent socioeconomic inequalities in shortterm MI case-fatality among men and women in Scotland between 1988 and 2004; inequalities for case-fatality during day 1-27 were comparatively smaller, but equally persistent. Igland et $a l^{10}$ reported educational inequalities in both 28 days and 29-365 days MI mortality that were strong and persistent during 2001-2009, even after adjustment for comorbidities and revascularisation. Yang et $a l^{8}$ found SEP inequalities in incidence and case-fatality after MI in Sweden between 1987 and 2008; the authors also report persistent SEP inequalities in MI incidence, similar to what we have reported previously, ${ }^{25}$ but did not address trends in case-fatality. While previous results are 
Table 4 Short-term and long-term case-fatality rates and $95 \%$ confidence limits per 1000 subjects for ischaemic stroke by socioeconomic position among men and women at prespecified ages 55, 65 and 75years old in 1990-1994 and 2005-2009

\begin{tabular}{|c|c|c|c|c|c|c|c|}
\hline & & Age & Low manual & High manual & Self-employed & Low non-manual & High non-manual \\
\hline \multicolumn{8}{|c|}{ Short-term case-fatality } \\
\hline \multirow[t]{4}{*}{ Men } & 1990-1994 & 55 & 63 (56 to 71$)$ & 61 (53 to 69) & 59 (51 to 68$)$ & 56 (48 to 65$)$ & 49 (42 to 55$)$ \\
\hline & & 75 & 134 (123 to 146$)$ & 130 (116 to 144$)$ & 127 (114 to 140$)$ & 121 (107 to 135$)$ & 106 (95 to 117) \\
\hline & 2005-2009 & 55 & 29 (25 to 33) & 27 (23 to 32) & 25 (21 to 29$)$ & 25 (21 to 29$)$ & 21 (18 to 24$)$ \\
\hline & & 75 & 75 (69 to 80$)$ & 70 (64 to 77$)$ & 65 (58 to 72) & 64 (58 to 70$)$ & 55 (51 to 60$)$ \\
\hline \multirow[t]{4}{*}{ Women } & 1990-1994 & 55 & 56 (49 to 63) & 54 (45 to 62$)$ & 52 (44 to 61$)$ & 50 (42 to 57$)$ & 43 (36 to 50$)$ \\
\hline & & 65 & 76 (68 to 84) & 73 (63 to 84) & 72 (61 to 82$)$ & 68 (59 to 77) & 59 (51 to 68$)$ \\
\hline & & 75 & 120 (108 to 131$)$ & 115 (100 to 131$)$ & 113 (99 to 127) & 107 (94 to 121$)$ & 94 (82 to 106) \\
\hline & 2005-2009 & 55 & 29 (25 to 33$)$ & 27 (23 to 32$)$ & 25 (21 to 30$)$ & 25 (21 to 29$)$ & 21 (18 to 25$)$ \\
\hline \multirow[t]{6}{*}{ Men } & 1990-1994 & 55 & 48 (42 to 56$)$ & 46 (39 to 54$)$ & 43 (37 to 50$)$ & 46 (39 to 54$)$ & 40 (35 to 47$)$ \\
\hline & & 65 & 76 (69 to 85$)$ & 73 (64 to 83 ) & 68 (60 to 78$)$ & 73 (64 to 83 ) & 64 (57 to 72 ) \\
\hline & & 75 & 151 (137 to 166$)$ & 144 (128 to 162$)$ & 135 (121 to 150$)$ & 144 (128 to 163$)$ & 127 (114 to 142$)$ \\
\hline & 2005-2009 & 55 & 39 (34 to 44$)$ & 35 (31 to 40 ) & 32 (28 to 37 ) & 35 (30 to 40 ) & 29 (25 to 33) \\
\hline & & 65 & 61 (57 to 67 ) & 55 (50 to 61$)$ & 50 (45 to 56$)$ & 55 (50 to 60$)$ & 46 (42 to 50$)$ \\
\hline & & 75 & 116 (109 to 124$)$ & 105 (97 to 113 ) & 96 (88 to 105) & 104 (96 to 113) & 87 (81 to 94) \\
\hline \multirow[t]{2}{*}{ Women } & 1990-1994 & 55 & 36 (31 to 42$)$ & 34 (28 to 41$)$ & 32 (27 to 38 ) & 34 (29 to 41$)$ & 30 (25 to 36$)$ \\
\hline & & 65 & 57 (51 to 64) & 54 (46 to 64$)$ & 51 (43 to 59$)$ & 54 (47 to 63$)$ & 48 (41 to 55$)$ \\
\hline
\end{tabular}

Age: prespecified ages 55, 65, 75 years old at diagnosis. Short-term case-fatality is death within 1 day including death outside the hospital. Long-term case-fatality is death within 1 year conditional on surviving the first day.

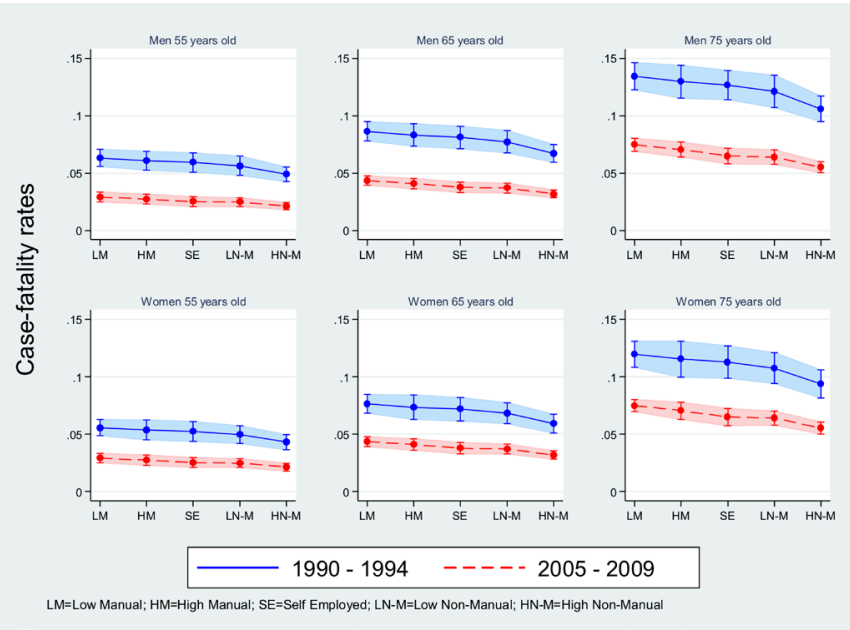

Figure 2 Short-term case-fatality rates in ischaemic stroke by socioeconomic position among men and women at prespecified ages 55, 65 and 75 years old in 1990-1994 and 2005-2009. in general agreement with our findings on MI, they are based on less complete information on short-term case-fatality (deaths outside the hospital were excluded), ${ }^{10}$ less precise SEP measurements (derived from geographical area), ${ }^{9}$ and less stringent distinction between short-term and long-term case-fatality (excluding all deaths within 28 days $)^{8}$ compared with our study.

Globally, almost $80 \%$ of mortality from ischaemic heart disease and $70 \%$ of mortality from stroke is attributed to physiological and behavioural risk factors including high blood pressure, high serum cholesterol, smoking, high body mass index, alcohol use, low intake of fruits and vegetables and physical inactivity. ${ }^{26}$ Sweden, as most other high-income countries, has benefited from decreasing trends in blood pressure, cholesterol levels, smoking and alcohol use, although these positive trends have partially been offset by rising obesity and glucose levels. ${ }^{27}$

Historically, there is clear evidence for SEP inequalities for these risk factors in Sweden during our earlier study period $1990-1994 .^{28}$ Recent studies, however, indicate 
that some of these differences have at least partially abated: SEP inequalities in both blood pressure levels as well as awareness and control of high blood pressure have decreased since then. ${ }^{29-31}$ Educational inequalities with regard to cholesterol levels were stable in men, but decreased among women, and access to treatment with lipid-lowering drugs did not differ between sex or educational levels. ${ }^{32}$ In the same period, educational inequalities in smoking and alcohol use have decreased in men, but increased in women. ${ }^{33}$ On the other hand, while a recent review suggested that the association between socioeconomic inequalities and obesity has remained stable at least until the beginning of our later study period 2005-2009,,$^{34}$ socioeconomic inequalities in leisure time physical inactivity have generally increased during this period. ${ }^{35}$

\section{Short-term case-fatality}

For both MI and IS, most cases of short-term case-fatality in our material are identified through CDR, limiting the potential role of improved clinical treatments in reducing short-term CFR. Determining factors for short-term survival are more generally disease severity and access to emergency acute care: the observed general decreases in short-term CFR may be attributed to a reduced severity of MI and IS due to overall positive trends in physiological and lifestyle factors as outlined above, as well as better management of pre-CVD risk factors (eg, use of angiotensin-converting enzyme inhibitors, aspirin and statins) or greater awareness of early symptoms and improvements in acute care treatment. ${ }^{67112436} 37$

\section{Myocardial infarction}

Assuming that the changes in short-term CFRD for MI at least partly reflect corresponding changes in risk factors, a possible explanation for the observed changes would be that the intermediate SEP groups at younger ages have embraced similar physiological and behavioural risk profiles for MI as the least disadvantaged HN-M group during the most recent study period 2005-2009, whereas the more disadvantaged LM group did not, at least not fully or in a manner where negative trends (like for physical leisure time activity) compensated for potential benefits due to positive trends (like, eg, for smoking and alcohol use).

\section{Ischaemic stroke}

Since high blood pressure is well-established as the main risk factor for all stroke subtypes,$^{38}$ the decrease in shortterm CFRD for IS seen in our study could be attributed to improved and more equitable control of high blood pressure among different SEP groups reducing incidence and severity of the IS events. Indeed, a Swedish study ${ }^{29}$ observed improved overall awareness and control of high blood pressure as well as diminishing differences in awareness and control between educational levels from 1990 to 2010.

\section{Long-term case-fatality}

Long-term case-fatality is affected by both the severity of the disease and quality of hospital care, including all previously mentioned physiological and behavioural risk factors for CVD that also affect short-term case-fatality as well as revascularisation and secondary care. A recent study presenting inequalities in secondary prevention after CVD in 21 countries reported Sweden as one of the countries providing most equitable access to the relevant drugs (antiplatelet, cholesterol and blood-pressure-lowering). ${ }^{39}$ It has, however, been indicated that treatment intensity at coronary care units after first MI is lower for men with low compared with high educational level. ${ }^{40}$ Other studies have also found evidence that statin medication is given preferentially to patients with a higher SEP after stroke ${ }^{41}$ or MI. ${ }^{42}$ In addition, patients with lower income have been reported as receiving revascularisation procedures to a lesser extent than patients with higher income. ${ }^{43}$

\section{Myocardial infarction}

We found that long-term CFRDs have increased in women, but not in men. While men have previously been reported to be almost twice as likely to undergo revascularisation procedures than women, the same study also found significant socioeconomic inequalities in access to coronary artery bypass grafting in men, but not in women. ${ }^{44}$ Further studies on long-term CFRD for MI among different SEP groups may be required to shed light on the reasons for increased CFRD among women.

\section{Ischaemic stroke}

Oral anticoagulants, which are recommended for secondary prevention against stroke, have been reported to be less often prescribed to older age groups and for individuals with lower educational level. ${ }^{45}$ Moreover, adherence to statin treatment over 2 years after stroke was lower in older age groups. ${ }^{46}$ These inequalities in medication for elderly can partly explain the increase CFRD at age 75 seen in our study.

\section{Short-term versus long-term case-fatality}

Short-term case-fatality is sometimes used as a proxy for severity of disease, ${ }^{19}$ which in turn is affected by disease prevention, awareness of early symptoms and the emergency transport system. Long-term case-fatality, on the other hand, is affected by the severity of the disease and also by the clinical treatment following the event. Consequently, we hypothesise that the driving forces behind broadly similar changes in short-term and long-term CFRs (stable for MI and decreasing for IS) are physiological and behavioural risk factors, and not primarily access to care after an event. This, however, does not explain the increasing SEP inequalities in long-term CFR among older men and women after IS and among women after MI, where inequitable care after an event may well be a contributing factor. Given the rapidly ageing population 
in Sweden and other high-income countries, this is potentially an important challenge for health services.

\section{Strengths}

To our knowledge, this is the first study to analyse shortterm and long-term CFRD for MI and IS over time for an entire population, systematically including mortality both before and after hospitalisation. Other studies about socioeconomic inequalities in MI or stroke $\mathrm{CFR}^{10}{ }^{47-50}$ did not distinguish in their analysis between short-term and longterm CFR; consequently, reports of decreasing long-term CFR as in Refs. 7, 47 and 48 could be biased by improvements in short-term CFR. Our approach, which models short-term CFR separately, allows us to deal adequately and statistically correctly with the fact that almost $1 / 3$ of all deaths during the study period occurred within the first day after the event. By combining the results of the two separate models, we can still report the overall 1-year CFR (online supplementary figure S5). Our sensitivity analyses for 7-day, 28-day and 90-day CFR support the separation of short-term and long-term models (data not shown): after taking the excess short-term case-fatality into account, the estimated CFRs for 7 days, 28 days and 90 days do not differ substantially from the long-term CFRs pattern reported here.

\section{Limitations}

We only report findings for ages $45-84$ years at diagnosis. The lower limit was set to exclude early-onset cases that are likely etiologically different from cases later in life. The upper limit was imposed by incomplete coverage in the population register and declining reliability of the main diagnosis in the CDR. However, these exclusions amounted to only $6 \%$ of all events during the study period. We had no information on risk factors, preventing us from investigating any medication mechanism for the association between SEP and CFR for MI or IS. Moreover, we do not have data on hospital care treatment before and after the event, such as revascularisation. The registers also lack of information important determinants of case-fatality, such as vascular comorbidity, medication adherence, access to emergency medical services and rural/urban living setting. The rural/urban divide also affects SEP and is therefore a potential confounder for the association between SEP and case-fatality. The other determinants such as vascular comorbidity are largely on the causal pathway from SEP to case-fatality and have consequently less potential for confounding this association.

As the latest census information available to us was from 1990, misclassification of SEP will affect the 20052009 cohort more strongly than the 1990-1994 cohort. To examine the strength of the bias this can introduce, we performed a sensitivity analysis where we backdated the SEP for subjects diagnosed 1990-1994 to the 1980 census; this did not change our findings (data not shown). The Swedish NPR has nation-wide coverage only after 1987, which may lead to misclassification of outcomes if we included individuals with prior disease as their first primary diagnosis. However, already from 1983 the coverage of NPR was $88 \%$ (online supplementary methods) and therefore the misclassification is probably small and should not bias our results dramatically. Short-term case-fatality is largely based on the CDR, and incorrect information in the CDR will lead to misclassification of the short-term outcomes. While Johansson et al have reported $87 \%$ agreement for ischaemic heart disease as main underlying cause of death in death certificates (on which the CDR is based) and assessment of case summaries, it is unknown to which degree misclassification is differential between SEP groups; consequently, we cannot rule out some degree of bias in our estimates for the association between SEP and short-term case-fatality. Due to legal limitations, the linkage of our study population against population registers can no longer be updated beyond 2010, therefore we were limited to the current study period.

Healthcare in Sweden is largely tax-funded with a low out-of-pocket payment, a system that ensures equal access to healthcare services for everyone. Costs for health and medical care as a percentage of Sweden's gross domestic product are stable and on par with most other European countries. ${ }^{51}$

Sweden has low rates of 30-day mortality following hospital admissions for acute MI and stroke as in many European countries. Moreover, the mortality in CVD is similar to the most of European countries. ${ }^{52}$ From international contents, our results should be comparable to most European countries that offer a universal healthcare system.

\section{CONCLUSION}

Both short-term and long-term CFR for MI and IS improved between study periods. Notably, both men and women in the LM group had a lower short-term CFR in 20052009 compared with HN-M group in 1990-1994. Despite extensive improvements, long-term CFRD remained and increased for MI in women and for IS among elderly men and women. With our novel model approach based on comparisons between short-term and long-term CFRs, we speculate that behavioural and physiological risk factors affecting MI and IS severity are the driving factors behind reduced SEP inequality in CFRs rather than more equitable access to hospital care, although continued research is required.

\section{Author affiliations}

${ }^{1}$ Department of Medical Epidemiology and Biostatistics, Karolinska Institutet, Stockholm, Sweden

${ }^{2}$ Centre of Clinical Research Sörmland, Uppsala University, Eskilstuna, Sweden ${ }^{3}$ Department of Public Health and Caring Sciences, Uppsala Universitet, Uppsala, Sweden

${ }^{4}$ Centre for Health Equity Studies, Stockholm University/Karolinska Institutet, Stockholm, Sweden

Contributors NM and PS conceived the original research idea. NM extracted the data. NM and AP conducted all data analyses and drafted the manuscript. NM, SH, 
ST, IK, PS and AP were involved in interpretation of results. NM, SH, ST, IK, PS and $A P$ revised the manuscript and agreed on the final version to be submitted.

Funding The authors have not declared a specific grant for this research from any funding agency in the public, commercial or not-for-profit sectors.

Competing interests None declared.

Patient consent for publication Not required.

Ethics approval Ethical approval (03-466) was obtained from the Regional Ethical Review Board in Stockholm, Sweden.

Provenance and peer review Not commissioned; externally peer reviewed.

Data sharing statement № additional data are available.

Open access This is an open access article distributed in accordance with the Creative Commons Attribution Non Commercial (CC BY-NC 4.0) license, which permits others to distribute, remix, adapt, build upon this work non-commercially, and license their derivative works on different terms, provided the original work is properly cited, appropriate credit is given, any changes made indicated, and the use is non-commercial. See: http://creativecommons.org/licenses/by-nc/4.0/.

\section{REFERENCES}

1. Lozano R, GBD 2013 Mortality and Causes of Death Collaborators. Global, regional, and national age-sex specific all-cause and causespecific mortality for 240 causes of death, 1990-2013: a systematic analysis for the Global Burden of Disease Study 2013. Lancet 2015;385:117-71.

2. Mackay J, Mensah G. The Atlas of Heart Disease and Stroke. 2004 http://www.who.int/cardiovascular_diseases/resources/atlas/en/\#.

3. Yeh RW, Sidney S, Chandra M, et al. Population trends in the incidence and outcomes of acute myocardial infarction. $N$ Engl J Med 2010;362:2155-65.

4. de Mestral C, Stringhini S. Socioeconomic Status and Cardiovascular Disease: an Update. Curr Cardiol Rep 2017;19:115.

5. Khaing W, Vallibhakara SA, Attia J, et al. Effects of education and income on cardiovascular outcomes: A systematic review and metaanalysis. Eur J Prev Cardiol 2017;24:1032-42.

6. Rosvall M, Gerward S, Engström G, et al. Income and short-term case fatality after myocardial infarction in the whole middle-aged population of Malmö, Sweden. Eur J Public Health 2008;18:533-8.

7. Chang WC, Kaul P, Westerhout CM, et al. Effects of socioeconomic status on mortality after acute myocardial infarction. Am J Med 2007;120:33-9.

8. Yang D, Dzayee DA, Beiki $O$, et al. Incidence and case fatality after day 28 of first time myocardial infarction in Sweden 1987-2008. Eur J Prev Cardiol 2012;19:1304-15.

9. Davies $\mathrm{CA}$, Leyland $\mathrm{AH}$. Trends and inequalities in short-term acute myocardial infarction case fatality in Scotland, 1988-2004. Popul Health Metr 2010;8:33.

10. Igland J, Vollset SE, Nygård OK, et al. Educational inequalities in 28 day and 1-year mortality after hospitalisation for incident acute myocardial infarction--a nationwide cohort study. Int J Cardiol 2014;177:874-80.

11. Parikh NI, Gona P, Larson MG, et al. Long-term trends in myocardial infarction incidence and case fatality in the National Heart, Lung, and Blood Institute's Framingham Heart study. Circulation 2009;119:1203-10.

12. Krumholz HM, Wang Y, Chen J, et al. Reduction in acute myocardial infarction mortality in the United States: risk-standardized mortality rates from 1995-2006. JAMA 2009;302:767-73.

13. The National Board of Health and Welfare. Statistikdatabasen. http:// www.socialstyrelsen.se/statistik/statistikdatabas2018

14. Sweden S. Socioekonomisk indelning: SEI = Swedish socioeconomic classification. Stockholm: Statistics Sweden, 1983.

15. Royston P, Lambert PC. Flexible parametric survival analysis using stata: beyond the Cox model. College Station, Tex.: Stata, 2011.

16. Royston P, Parmar MK. Flexible parametric proportional-hazards and proportional-odds models for censored survival data, with application to prognostic modelling and estimation of treatment effects. Stat Med 2002;21:2175-97.

17. Agresti A. Categorical data analysis. 3rd edition, 2013.

18. Berg J, Björck L, Nielsen S, et al. Sex differences in survival after myocardial infarction in Sweden, 1987-2010. Heart 2017;103:1625-30.

19. Dudas K, Lappas G, Stewart S, et al. Trends in out-of-hospital deaths due to coronary heart disease in Sweden (1991 to 2006). Circulation 2011;123:46-52.
20. Pennlert J, Asplund K, Glader EL, et al. Socioeconomic status and the risk of stroke recurrence: persisting gaps observed in a nationwide swedish study 2001 to 2012. Stroke 2017;48:1518-23.

21. Dudas K, Lappas G, Rosengren A. Long-term prognosis after hospital admission for acute myocardial infarction from 1987 to 2006. Int J Cardiol 2012;155:400-5.

22. Dégano IR, Salomaa V, Veronesi G, et al. Twenty-five-year trends in myocardial infarction attack and mortality rates, and case-fatality, in six European populations. Heart 2015;101:1413-21.

23. Nichols M, Townsend N, Scarborough P, et al. Cardiovascular disease in Europe 2014: epidemiological update. Eur Heart $J$ 2014;35:2950-9.

24. Feigin VL, Lawes CM, Bennett DA, et al. Worldwide stroke incidence and early case fatality reported in 56 population-based studies: a systematic review. Lancet Neurol 2009;8:355-69.

25. Malki N, Koupil I, Eloranta S, et al. Temporal trends in incidence of myocardial infarction and ischemic stroke by socioeconomic position in Sweden 1987-2010. PLoS One 2014;9:e105279.

26. Ezzati M, Hoorn SV, Rodgers A, et al. Estimates of global and regional potential health gains from reducing multiple major risk factors. Lancet 2003;362:271-80.

27. Kontis V, Mathers CD, Bonita R, et al. Regional contributions of six preventable risk factors to achieving the $25 \times 25$ non-communicable disease mortality reduction target: a modelling study. Lancet Glob Health 2015;3:e746-7.

28. Peltonen M, Huhtasaari F, Stegmayr B, et al. Secular trends in social patterning of cardiovascular risk factor levels in Sweden. The Northern Sweden MONICA Study 1986-1994. Multinationa Monitoring of Trends and Determinants in Cardiovascular Disease. $J$ Intern Med 1998;244:1-9.

29. $\mathrm{Ng} \mathrm{N}$, Carlberg B, Weinehall L, et al. Trends of blood pressure levels and management in Västerbotten County, Sweden, during 19902010. Glob Health Action 2012;5:18195.

30. Eriksson M, Carlberg B, Pennlert J, et al. Time trends and socioeconomic differences in blood pressure levels: The Northern Sweden MONICA study 1994-2014. Eur J Prev Cardiol 2017;24:1473-81.

31. Törmä E, Carlberg B, Eriksson M, et al. Long term trends in control of hypertension in the Northern Sweden MONICA study 1986-2009. BMC Public Health 2015;15:957.

32. Eriksson M, Forslund AS, Jansson JH, et al. Greater decreases in cholesterol levels among individuals with high cardiovascular risk than among the general population: the northern Sweden MONICA study 1994 to 2014. Eur Heart J 2016;37:1985-92.

33. Östergren $O$, Martikainen $P$, Lundberg $O$. The contribution of alcohol consumption and smoking to educational inequalities in life expectancy among Swedish men and women during 1991-2008. Int J Public Health 2018;63:41-8.

34. Magnusson M, Sørensen TI, Olafsdottir S, et al. Social inequalities in obesity persist in the nordic region despite its relative affluence and equity. Curr Obes Rep 2014;3:1-15.

35. Szilcz M, Mosquera PA, Sebastián MS, et al. Time trends in absolute and relative socioeconomic inequalities in leisure time physical inactivity in northern Sweden. Scand J Public Health 2018;46:112-23

36. Myerson M, Coady S, Taylor $\mathrm{H}$, et al. Declining severity of myocardial infarction from 1987 to 2002: the Atherosclerosis Risk in Communities (ARIC) Study. Circulation 2009;119:503-14.

37. Addo J, Ayerbe L, Mohan KM, et al. Socioeconomic status and stroke: an updated review. Stroke 2012;43:1186-91.

38. Lawes CM, Bennett DA, Feigin VL, et al. Blood pressure and stroke: an overview of published reviews. Stroke 2004;35:1024.

39. Murphy A, Palafox B, O'Donnell O, et al. Inequalities in the use of secondary prevention of cardiovascular disease by socioeconomic status: evidence from the PURE observational study. Lancet Glob Health 2018;6:e292-e301.

40. Yang D, James $S$, de Faire $U$, et al. Likelihood of treatment in a coronary care unit for a first-time myocardial infarction in relation to sex, country of birth and socioeconomic position in Sweden. PLOS One 2013;8:e62316.

41. Sjölander M, Eriksson M, Glader EL. Social stratification in the dissemination of statins after stroke in Sweden. Eur J Clin Pharmacol 2013;69:1173-80.

42. Ringbäck Weitoft G, Ericsson O, Löfroth E, et al. Equal access to treatment? Population-based follow-up of drugs dispensed to patients after acute myocardial infarction in Sweden. Eur J Clin Pharmacol 2008;64:417-24.

43. Rosvall M, Chaix B, Lynch J, et al. The association between socioeconomic position, use of revascularization procedures and five-year survival after recovery from acute myocardial infarction. BMC Public Health 2008;8:44 
44. Haglund B, Köster M, Nilsson T, et al. Inequality in access to coronary revascularization in Sweden. Scand Cardiovasc J 2004;38:334-9.

45. Sjölander M, Eriksson M, Asplund K, et al. Socioeconomic Inequalities in the Prescription of Oral Anticoagulants in Stroke Patients With Atrial Fibrillation. Stroke 2015;46:2220-5.

46. Sjölander M, Eriksson M, Glader EL. Inequalities in medication adherence to statin treatment after stroke: A nationwide observational study. Eur Stroke J 2016;1:101-7.

47. Bergström $\mathrm{G}$, Redfors $\mathrm{B}$, Angerås $\mathrm{O}$, et al. Low socioeconomic status of a patient's residential area is associated with worse prognosis after acute myocardial infarction in Sweden. Int $J$ Cardiol 2015;182:141-7.

48. Salomaa V, Niemelä M, Miettinen $\mathrm{H}$, et al. Relationship of socioeconomic status to the incidence and prehospital, 28- day, and 1-year mortality rates of acute coronary events in the FINMONICA myocardial infarction register study. Circulation 2000;101:1913-8.

49. Fornari C, Cesana GC, Chambless LE, et al. Time trends of myocardial infarction 28-day case-fatality in the 1990s: is there a contribution from different changes among socioeconomic classes? $J$ Epidemiol Community Health 2008;62:593-8.

50. Lammintausta A, Immonen-Räihä P, Airaksinen JK, et al. Socioeconomic inequalities in the morbidity and mortality of acute coronary events in Finland: 1988 to 2002. Ann Epidemiol 2012;22:87-93.

51. Aanestad M, Grisot M, Hanseth $\mathrm{O}$, et al. Information infrastructures within european health care, 2017.

52. Wilkins E, Wilson L, Wickramasinghe K, et al. European cardiovascular disease statistics 2017. Brussels, 2017. 